\title{
Effect of Coulomb correlation on charge transport in disordered organic semiconductors
}

\author{
Feilong Liu, ${ }^{1, *}$ Harm van Eersel, ${ }^{2}$ Bojian Xu ${ }^{3}$ Janine G. E. Wilbers, ${ }^{3}$ Michel P. de Jong, ${ }^{3}$ \\ Wilfred G. van der Wiel, ${ }^{3}$ Peter A. Bobbert, ${ }^{1,3,4}$ and Reinder Coehoorn ${ }^{1,4}$ \\ ${ }^{1}$ Department of Applied Physics, Eindhoven University of Technology, P.O. Box 513, 5600 MB Eindhoven, The Netherlands \\ ${ }^{2}$ Simbeyond B.V., P.O. Box 513, 5600 MB Eindhoven, The Netherlands \\ ${ }^{3}$ NanoElectronics Group, MESA+ Institute for Nanotechnology, University of Twente, P.O. Box 217, 7500 AE Enschede, The Netherlands \\ ${ }^{4}$ Institute for Complex Molecular Systems, Eindhoven University of Technology, P.O. Box 513, 5600 MB Eindhoven, The Netherlands
}

(Received 1 September 2017; revised manuscript received 16 October 2017; published 20 November 2017)

\begin{abstract}
Charge transport in disordered organic semiconductors, which is governed by incoherent hopping between localized molecular states, is frequently studied using a mean-field approach. However, such an approach only considers the time-averaged occupation of sites and neglects the correlation effect resulting from the Coulomb interaction between charge carriers. Here, we study the charge transport in unipolar organic devices using kinetic Monte Carlo simulations and show that the effect of Coulomb correlation is already important when the charge-carrier concentration is above $10^{-3}$ per molecular site and the electric field is smaller than $10^{8} \mathrm{~V} / \mathrm{m}$. The mean-field approach is then no longer valid, and neglecting the effect can result in significant errors in device modeling. This finding is supported by experimental current density-voltage characteristics of ultrathin sandwich-type unipolar poly(3-hexylthiophene) (P3HT) devices, where high carrier concentrations are reached.
\end{abstract}

DOI: 10.1103/PhysRevB.96.205203

\section{INTRODUCTION}

In the past decades, the understanding of charge transport in disordered organic semiconductors has greatly increased [1-4]. Assuming incoherent hopping of charge carriers between localized states and using advanced three-dimensional (3D) mechanistic modeling techniques, it is now possible to predict the macroscopic charge transport properties of organic semiconductors using microscopic information at the molecular level, including a Gaussian distribution of site energies, the spatial packing of the material, the distribution of charge transfer integrals, and the reorganization energies [512]. Device modeling has been carried out using drift-diffusion [13-18], master-equation (ME) [19-23], and kinetic Monte Carlo (KMC) [2,24-28] simulation methods. Within driftdiffusion and $\mathrm{ME}$ device simulations, the Coulomb interaction between the charge carriers is treated using a mean-field approach: using the Poisson equation, the (time-averaged) charge density is used to calculate the (time-averaged) electric field due to the space charge in the device. Only with 3D KMC simulations, the Coulomb correlation between the individual charge carriers can be explicitly taken into account.

Both experimental and theoretical work has shown that in organic field-effect transistors Coulomb correlation can, at large gate voltages, give rise to a decrease of the field-effect mobility with increasing gate voltages [29,30]. Theoretical work indicates that at low temperature and for high carrier densities, Coulomb correlation in disordered materials can give rise to various interesting phenomena, such as the formation of a "Coulomb glass" and ultraslow relaxation effects [31]. In these cases, KMC simulations can provide helpful insights. KMC simulations are also helpful when studying bipolar devices: the process of exciton formation is then in a parameterfree and mechanistic way included [32]. When studying unipolar devices, the advantage of using KMC simulations, which

\footnotetext{
*f.liu@tue.nl
}

are computationally more demanding than drift-diffusion or ME simulations, is not immediately obvious. The question arises to what extent, in the case of transport in unipolar sandwich-type devices, mean-field approaches are sufficiently accurate. Cottaar et al. [33] showed that due to charge accumulation near interfaces, Coulomb correlation can indeed be important in unipolar devices. On the other hand, for the case of bulk transport in materials with a Gaussian density of states (DOS), Zhou et al. concluded from KMC simulations that the mean-field approach may be used up to a charge concentration of $10^{-2}$ per site [24]. However, in that work only transport in a relatively large electric field $\left(10^{8} \mathrm{~V} / \mathrm{m}\right)$ was studied, not answering the question what would happen in smaller fields.

In this paper, we show that at lower electric fields $\left(10^{6}-10^{7} \mathrm{~V} / \mathrm{m}\right)$, which are relevant to realistic operational conditions in, e.g., organic light-emitting diodes (OLEDs), Coulomb correlation can significantly reduce the chargecarrier mobility in materials with a Gaussian DOS. From a comparison between $\mathrm{ME}$ and $\mathrm{KMC}$ simulation results, it follows that at a concentration of $10^{-3}$ charge carriers per site the mobility reduction can already be a factor of 3 or larger for materials with realistic energetic disorder. The competition between the Coulomb field due to the charge-charge interaction and the external electric field leads to an enhanced field dependence of mobility. The charge-carrier concentration dependence of the mobility is significantly reduced by the Coulomb correlation. At large carrier concentrations and weak disorder, even a distinct decrease of the mobility with increasing carrier density is found.

The paper is organized as follows. In Sec. II, we describe the ME (mean-field) and KMC (beyond-mean-field) simulation methods used. In Sec. III, we compare the charge-carrier mobility calculated using both methods for systems with a spatially uniform external field. In Sec. IV, we perform full device simulations and compare the current density-voltage characteristics and charge-carrier concentration profiles calculated from ME and KMC modeling. Due to the Coulomb correlation, a significant difference is found between the 
two modeling approaches using the same set of material parameters. Subsequently, experimental current density-voltage characteristics for a series of unipolar poly(3-hexylthiophene) (P3HT) devices with various thicknesses and corresponding modeling results are considered, providing an example of a system for which Coulomb correlation is highly relevant. In Sec. V, we present a summary and our conclusions.

\section{METHODS}

The organic semiconductor structure is described as a 3D cubic lattice with a lattice constant $a$, representing the actual molecular sites. The site energies are randomly taken from a Gaussian distribution with a width (standard deviation) $\sigma$. Charge carriers hop incoherently between neighboring sites, with a distance and energy difference dependence as given by the Miller-Abrahams formula [34]. This formalism includes the probability that hops occur over a large distance. However, when considering transport at room temperature in singlecomponent systems with realistic values of $\sigma$ in the range 0.05 to $0.15 \mathrm{eV}$ and for small values of the effective wave function decay length $(\lambda \simeq 0.1 \mathrm{~nm})$, the average hop distance is not much larger than the lattice constant $a$ [21,35]. For simplicity, we consider in Secs. III and IV A only nearestneighbor hopping, but include variable-range hopping in Sec. IV B when comparing with experimental results. We checked that varying $\lambda$ does not change the conclusion of this work.

The KMC simulations are carried out using the Bumblebee tool [36], within which the method that has been described in Ref. [25] is implemented. When carrying out device simulations, the systems studied are rectangular boxes consisting of a number of molecular layers corresponding to the actual device thickness considered. The boxes have equal dimensions in the two lateral directions, to which periodic boundary conditions are applied, and are bounded in the third dimension by the metallic electrodes at which carrier injection and collection takes place. To simulate the dynamics of individual charge carriers, the hopping rates of each carrier are calculated after including (i) the effect of the potential due to a uniform applied external field, (ii) the 3D Coulomb potential due to all individual other charge carriers within a certain sphere with a cutoff radius $R_{\mathrm{c}}$ [25], (iii) the Coulomb potential due to the layer-averaged charge density outside the cutoff sphere, as obtained by solving the Poisson equation, and (iv) the image potential due to the presence of the two metallic electrodes. $R_{\mathrm{c}}$ is taken to be large enough, such that a further increase of $R_{\mathrm{c}}$ does not affect the results (see Sec. III). When using $\mathrm{KMC}$ simulations for calculating the mobility in the presence of a uniform external field and for a spatially uniform average charge-carrier concentration, the system studied is a cubic box with periodic boundary conditions in all three directions. The electrostatic potential that determines the hopping rate of each carrier is then only determined by the contributions from all other individual carriers within the cutoff radius and by the potential due to the uniform external field.

Similarly, boxes with periodic boundary conditions in two or three directions were considered when carrying out $\mathrm{ME}$ device simulations and mobility studies, respectively. The Coulomb interaction is then treated in a mean-field way, which is equivalent to the case of KMC simulations with $R_{\mathrm{c}}=0$.
The self-consistent steady-state occupational probability at all molecular sites is obtained numerically. Subsequently, the current density is calculated by summing the products of all hopping rates multiplied by the corresponding site occupational probabilities $[21,22]$. The image-charge effect is taken into account using a method that most accurately mimics the results obtained from KMC simulations [22].

All simulations are continued until steady state conditions are reached, and (in the case of KMC simulations) until the mean (time-averaged) current density can be obtained with a sufficient statistical accuracy. The results do not depend on the initial conditions such as the initial distribution of charge carriers in the system. To avoid finite size effects when treating disordered systems, the simulation box sizes need to be sufficiently large [37]. For device simulations, we use boxes containing approximately $1.6 \times 10^{5}$ sites (such that for thinner devices the lateral box dimensions are correspondingly larger). For simulations with periodic boundary conditions in all three directions we use cubic boxes with $10^{6}-3 \times 10^{6}$ sites. The calculated mobility and current density values are averaged over typically $5-10$ boxes with different disorder configurations.

\section{CHARGE-CARRIER MOBILITY}

\section{A. Simulation results}

In this section, we study the Coulomb correlation effect on the charge-carrier mobility for a Gaussian energy disorder, using parameter values that are typical for disordered organic semiconductors $[5,9,21,22]$. For all systems, $a$ is taken equal to be $1 \mathrm{~nm}$. The hopping rate to a nearest-neighbor site with an equal site energy, $v_{1}$, is taken equal to $3.3 \times 10^{10} \mathrm{~s}^{-1}$. We first focus on systems with a fixed relative dielectric constant, $\varepsilon_{\mathrm{r}}=3$, studied at a temperature $T=290 \mathrm{~K}$. The electric field $F$ is varied from $10^{6} \mathrm{~V} / \mathrm{m}$ to $10^{8} \mathrm{~V} / \mathrm{m}$ and the charge-carrier concentration $c$ is varied from $10^{-5}$ to $10^{-2}$ carriers per site. The values of the energetic disorder strength considered are $\sigma=0.05,0.10$, and $0.15 \mathrm{eV}$, corresponding to values $\sigma /\left(k_{\mathrm{B}} T\right)$ that are approximately equal to 2,4 , and 6 , respectively. Here $k_{\mathrm{B}}$ is the Boltzmann constant. The size of the simulation box is $150 \times 150 \times 150$ for $c=10^{-5}$ and $10^{-4}$, and $100 \times 100 \times 100$ for $c=10^{-3}$ and $10^{-2}$. Within the KMC simulations, the Coulomb cutoff radius $R_{\mathrm{c}}$ is taken to be $15 \mathrm{~nm}$ for $c=10^{-5}, 10^{-4}$, and $10^{-3}$, and $10 \mathrm{~nm}$ for $c=10^{-2}$. With these values, accurate and converged results are obtained. The mobility values from ME and KMC simulations are extracted following the methods in Refs. [21,24].

Figure 1 shows the charge-carrier mobility as a function of the electric field, at various charge-carrier concentrations and disorder strengths, as obtained from ME (solid curves) and KMC (symbols) simulations. We find that well below $c=10^{-4}$ the mobilities as obtained from the ME and KMC simulations agree very well and conclude that the mean-field approach is then valid (see Fig. 2). However, the Coulomb correlation leads for $c=10^{-4}$ at low fields already to an observable reduction of the mobility. For $c=10^{-3}$, the mobility reduction can be as large as a factor 3 or even more, while for $c=10^{-2}$ the mobility reduction at low fields ranges from a factor of about 30 for $\sigma /\left(k_{\mathrm{B}} T\right)=6$ to more than a factor 300 for $\sigma /\left(k_{\mathrm{B}} T\right)=2$. 


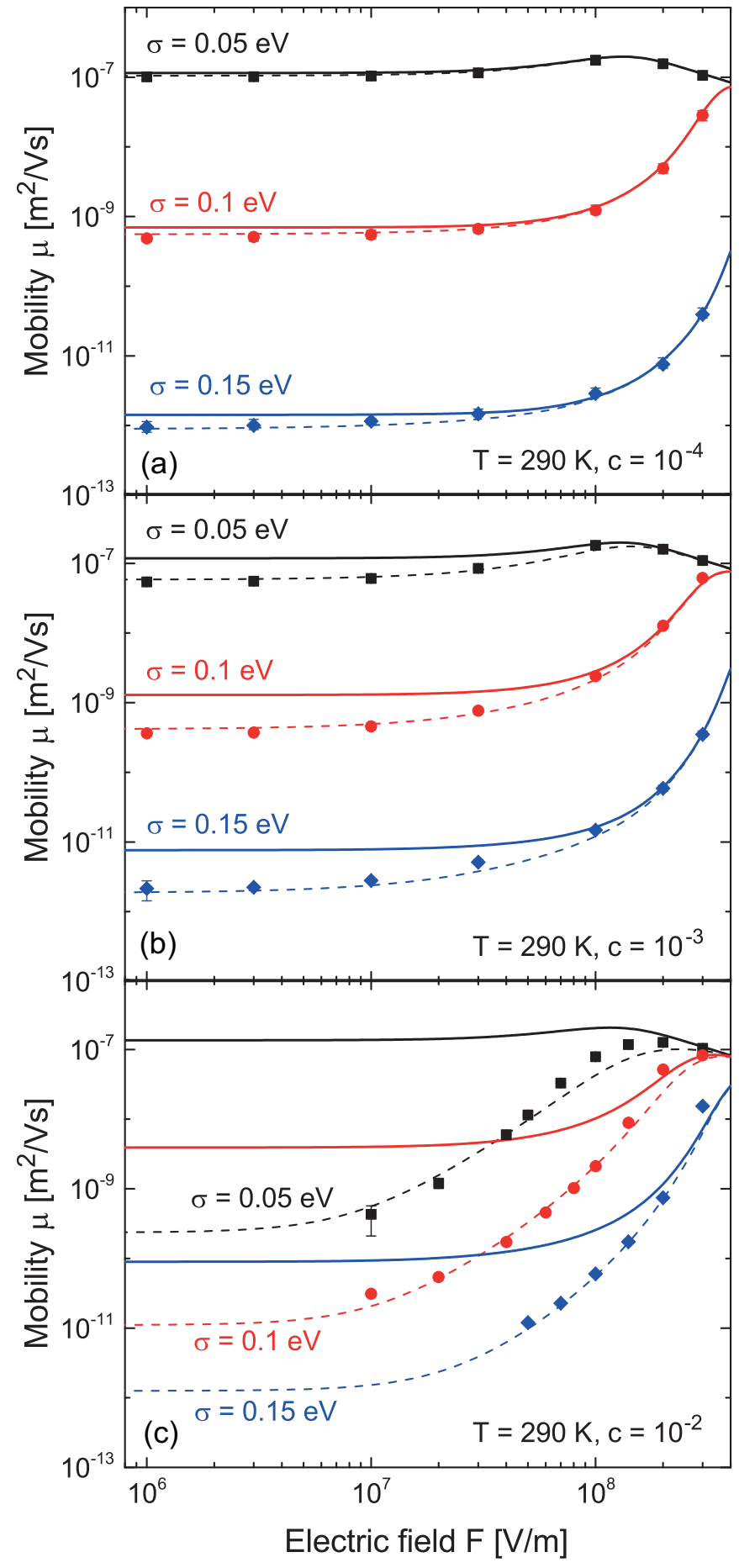

FIG. 1. Calculated charge-carrier mobility $\mu$ as a function of the electric field $F$, at various charge-carrier concentrations $c$ and disorder strengths $\sigma$, for relative dielectric constant $\varepsilon_{\mathrm{r}}=3$ and $T=290 \mathrm{~K}$. Solid curves: ME results $\left(\mu_{\mathrm{ME}}\right)$. Symbols: KMC results ( $\left.\mu_{\mathrm{KMC}}\right)$. Dashed curves: $\mu_{\mathrm{ME}}$ multiplied by the empirical mobility reduction factor given by Eqs. (1)-(3).

The mobility reduction decreases as the electric field increases, so that the field dependence of the mobility is enhanced by Coulomb correlation. When the external field dominates over the Coulomb field, the mobility reduction due to Coulomb correlation is small. Consistent with the results of Zhou et al. [24], the mobility reduction is very small at $F=10^{8} \mathrm{~V} / \mathrm{m}$.

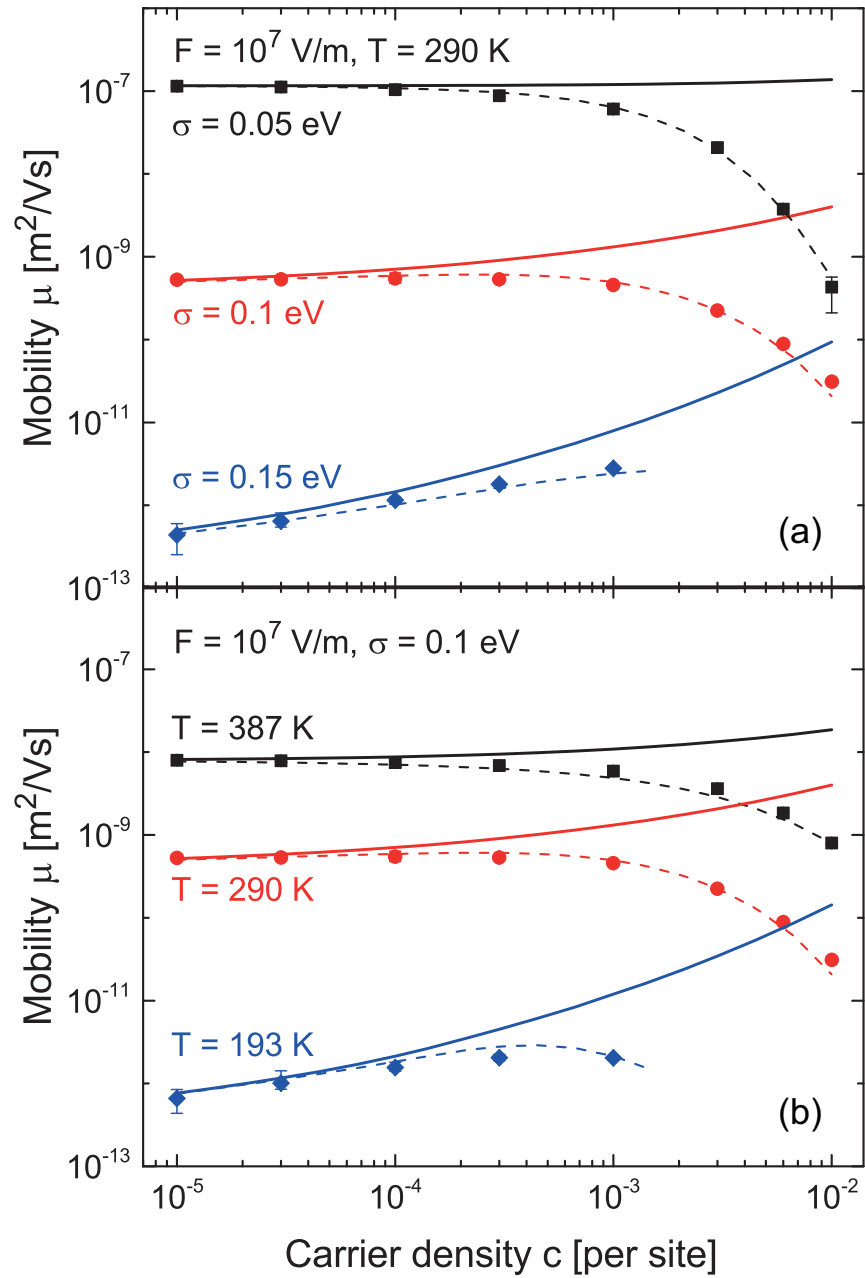

FIG. 2. Calculated charge-carrier mobility $\mu$ as a function of the carrier concentration $c$, at various disorder strengths $\sigma$ (a) or temperature $T$ (b), for $F=10^{7} \mathrm{~V} / \mathrm{m}$ and relative dielectric constant $\varepsilon_{\mathrm{r}}=3$. Solid curves: ME results $\left(\mu_{\mathrm{ME}}\right)$. Symbols: KMC results $\left(\mu_{\mathrm{KMC}}\right)$. Dashed curves: $\mu_{\mathrm{ME}}$ multiplied by the empirical mobility reduction factor given by Eqs. (1)-(3).

At $F=2 \times 10^{8} \mathrm{~V} / \mathrm{m}$, the effect is almost negligible. For $c=$ $10^{-2}$ and small electric fields, the system behaves in the KMC simulations to some extent similarly to a strongly Coulombcorrelated system ("Coulomb glass") [38-41]: the convergence to a well-defined thermal equilibrium state becomes very slow. We are, at this high carrier concentration, therefore unable to obtain converged results for electric fields below $10^{7} \mathrm{~V} / \mathrm{m}$.

At low fields, the Coulomb correlation strongly affects the carrier concentration dependence of the mobility. This may be seen from Fig. 2, which shows for three values of the disorder strength and temperature the carrier concentration dependence of the mobility at a field $F=10^{7} \mathrm{~V} / \mathrm{m}$. The mobility increases outside the low-concentration independent-particle (Boltzmann) regime with increasing carrier concentration due to the filling of deep states that would otherwise act as traps. However, at low fields this effect is strongly reduced for the KMC results due to the Coulomb correlation, and for systems with a small disorder even a distinct decrease of the mobility is found.

The reduction of the mobility due to the Coulomb correlation is found to depend strongly on the relative dielectric 


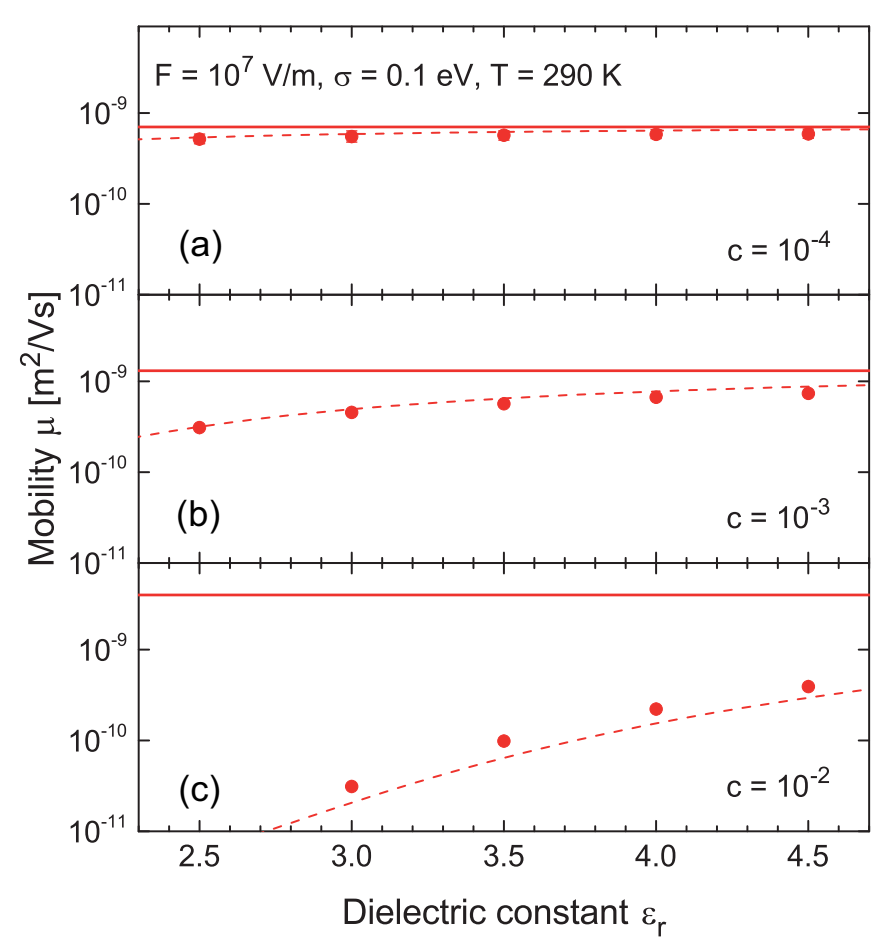

FIG. 3. Calculated charge-carrier mobility $\mu$ as a function of the relative dielectric constant $\varepsilon_{\mathrm{r}}$ at various carrier concentrations $c$, for $\sigma=0.1 \mathrm{eV}, F=10^{7} \mathrm{~V} / \mathrm{m}$, and $T=290 \mathrm{~K}$. Solid curves: ME results $\left(\mu_{\mathrm{ME}}\right)$. Symbols: KMC results $\left(\mu_{\mathrm{KMC}}\right)$. Dashed curves: $\mu_{\mathrm{ME}}$ multiplied by the empirical mobility reduction factor given by Eqs. (1)-(3).

constant $\varepsilon_{\mathrm{r}}$. Figure 3 shows the dependence of the mobility on $\varepsilon_{\mathrm{r}}$ for systems with various values of the carrier concentration, for $\sigma=0.1 \mathrm{eV}, F=10^{7} \mathrm{~V} / \mathrm{m}$, and $T=290 \mathrm{~K}$. For the largest carrier concentration considered $\left(c=10^{-2}\right)$, the mobility varies over more than one order of magnitude when $\varepsilon_{\mathrm{r}}$ is varied in the range $2.5-4.5$. We have verified that in the limit of $\varepsilon_{\mathrm{r}} \rightarrow \infty$ (where Coulomb correlation vanishes), the ME and KMC approaches yield identical results.

\section{B. Parameterization scheme}

When a mean-field approach is applicable, the mobility function may be written as the product of the mobility in the limit of zero carrier concentration, zero field and infinite temperature, $\mu_{0}$, multiplied by a universal function that only depends on reduced (dimensionless) parameters: the carrier concentration $c$, the relative disorder strength $\hat{\sigma}=\sigma /\left(k_{\mathrm{B}} T\right)$, and the reduced field $\hat{F}=e a F / \sigma$ [21]. The universal mobility function may then already be determined by studying the mobility for a single value of the material parameters $a$ and $\sigma$. We note that the latter only holds for the case of transport dominated by nearest-neighbor hopping considered in the present section. When variable-range hopping becomes dominant, the wave function decay length $\lambda$ enters as a parameter in the field dependence [42].

For systems in which Coulomb correlation is significant, $\varepsilon_{\mathrm{r}}$ becomes a relevant material parameter. We follow an empirical approach that aims at providing a fair description of the reduction of the mobility due to the Coulomb correlation, using a minimal set of dimensionless parameters.

We find that the following parametrization scheme provides a fair description of the ratio of the mobility $\mu_{\mathrm{KMC}}$ as obtained from the KMC approach, which includes the effect of Coulomb correlation, and the mobility $\mu_{\mathrm{ME}}$ as obtained from the ME approach:

$$
\frac{\mu_{\mathrm{KMC}}(c, T, F)}{\mu_{\mathrm{ME}}(c, T, F)}=1-f_{1}(c, T, F) f_{2}(c, T),
$$

with

$$
f_{1}(c, T, F)=\exp \left[-\left(\frac{|F|}{2 F_{0} c^{\frac{1}{3}} \frac{\varepsilon_{\mathrm{r}}}{3}}\right)^{\left(1+\frac{c}{0.01} \frac{290}{T}\right)}\right]
$$

and

$$
f_{2}(c, T)=\tanh \left\{\frac{3}{\varepsilon_{\mathrm{r}}}\left[\left(1+0.5 \frac{\sigma}{k_{\mathrm{B}} T}\right) \frac{c}{c_{0}}\right]^{\left(\frac{290}{T}-0.1 \frac{\sigma}{k_{\mathrm{B}} T}\right)}\right\} .
$$

Here, $F_{0}=e /\left(4 \pi \varepsilon_{0} \varepsilon_{\mathrm{r}} a^{2}\right)$ is the electric field at a distance $a$ from a unit charge, and $c_{0}=\varepsilon_{0} \varepsilon_{\mathrm{r}} k_{\mathrm{B}} T a / e^{2}$ is the carrier concentration at which the Debye screening length is equal to $a$. The function $f_{1}$ contains the normalized electric field dependence of the mobility reduction, and the function $f_{2}$ contains the relative mobility reduction in the limit of zero electric field, as a function of the carrier concentration. Because of the complexity of the effects of Coulomb correlation, the carrier concentration and temperature dependence are not fully separable, and both variables $c$ and $T$ appear in both $f_{1}$ and $f_{2}$. Consistent with the results in Fig. 1 , the function $f_{1}$ describes an $s$-shape dependence on the electric field.

In Figs. 1-3, the mobility as obtained using this parametrization scheme is indicated by the dashed curves. Although, for the cases studied, the comparison with the KMC simulation data is good, further testing by more extensively varying the simulation parameters is needed, because the mobility reduction does not simply depend on the relative disorder strength $\hat{\sigma}$ and the reduced field $\hat{F}$ (Fig. 2). The results of such a study are given in figures S1-S4 of the Supplemental Material [43]. In Fig. S1, a comparison is given between the simulated field dependence of the mobility obtained (1) at $290 \mathrm{~K}$, for disorder strengths $\sigma=0.075,0.10$, and $0.15 \mathrm{eV}$, and (2) at disorder strength $\sigma=0.10 \mathrm{eV}$, for temperatures $T=387,290$, and $193 \mathrm{~K}$. The simulations are thus in both cases carried out for relative disorder strengths $\hat{\sigma}=3,4$, and 6 , as realized by either varying the disorder strength or the temperature. This figure shows that these two ways of varying the relative disorder strength lead to a distinctly different field dependence of the mobility, confirming that $c, \hat{\sigma}$, and $\hat{F}$ are no longer the only relevant dimensionless parameters when the Coulomb interaction is included. Fig. S2 gives a similar comparison for the carrier concentration dependence of the mobility. Also in this case, clear deviations from a dependence on only $\hat{\sigma}$ are apparent. The range of parameters for which the $\varepsilon_{\mathrm{r}}$-dependence of the mobility has been studied is extended in Fig. S3 by including the dependence on $\sigma$, and in Fig. S4 by including the dependence on $T$, in both cases for two values of $F$. For all these additional cases, the parametrization scheme is found to provide a fair description of the simulation data. 


\section{CURRENT DENSITY IN DEVICES}

\section{A. The Coulomb correlation effect in unipolar sandwich-type devices}

In this section, we investigate the impact of Coulomb correlation on the current density and charge-carrier density in unipolar sandwich-type devices. We focus on the transport at $290 \mathrm{~K}$ through a single layer of an organic semiconductor with $\sigma=0.1 \mathrm{eV}$ and $\varepsilon_{\mathrm{r}}=3$, in devices with a varying thickness $(L)$ and a varying injection barrier $(\Phi)$ that is equal at the two electrode interfaces. Figure 4 gives the ratio $J_{\mathrm{KMC}} / J_{\mathrm{ME}}$ of the current densities as obtained from $\mathrm{KMC}$ and $\mathrm{ME}$ simulations. This ratio gives the reduction of the current density obtained with the KMC approach, which includes Coulomb correlation, with respect to current density obtained from the ME approach. Because recently a technique has been developed for fabricating devices with organic layer thicknesses as small as $5 \mathrm{~nm}$ (see the next subsection), simulation results down to that thickness have been included in the figure. We note that the devices with zero injection barrier required a particularly strong simulation effort, because a very large fraction of the total number of Monte Carlo steps involves charge injection and collection events at the interface between one of the electrodes and the adjacent organic layer, yielding hardly any net contribution to the current density.

For the devices with the largest injection barrier considered $(0.4 \mathrm{eV})$, the simulations show that Coulomb correlation is for almost all cases studied negligible. This may be understood from the small carrier concentration in the bulk of the devices, which results from the large injection barrier. As an example, Fig. 5(a) shows the charge-carrier concentration profile as obtained from the ME and KMC simulations for

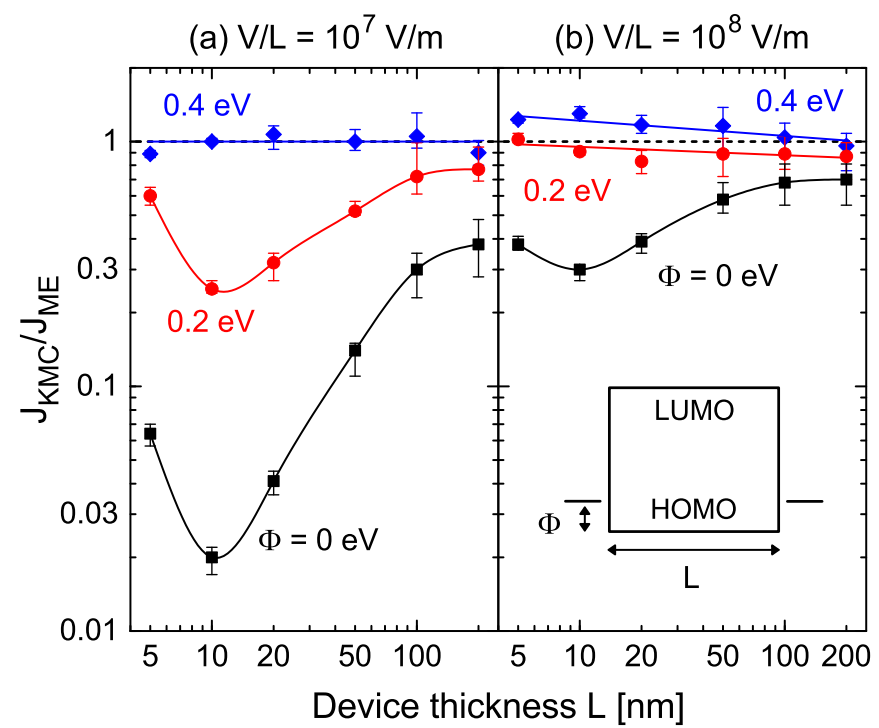

FIG. 4. Ratio $J_{\mathrm{KMC}} / J_{\mathrm{ME}}$ of the current densities $J_{\mathrm{KMC}}$ and $J_{\mathrm{ME}}$ as obtained from KMC and ME simulations, respectively, for symmetric unipolar sandwich-type devices at various device thicknesses $L$ and injection barriers $\Phi$, for $V / L=10^{7} \mathrm{~V} / \mathrm{m}$ (a) and $V / L=$ $10^{8} \mathrm{~V} / \mathrm{m}$ (b), respectively. The devices contain a single layer of a semiconductor with a Gaussian DOS of width $\sigma=0.1 \mathrm{eV}$ and with $\varepsilon_{\mathrm{r}}=3$, studied at $T=290 \mathrm{~K}$.
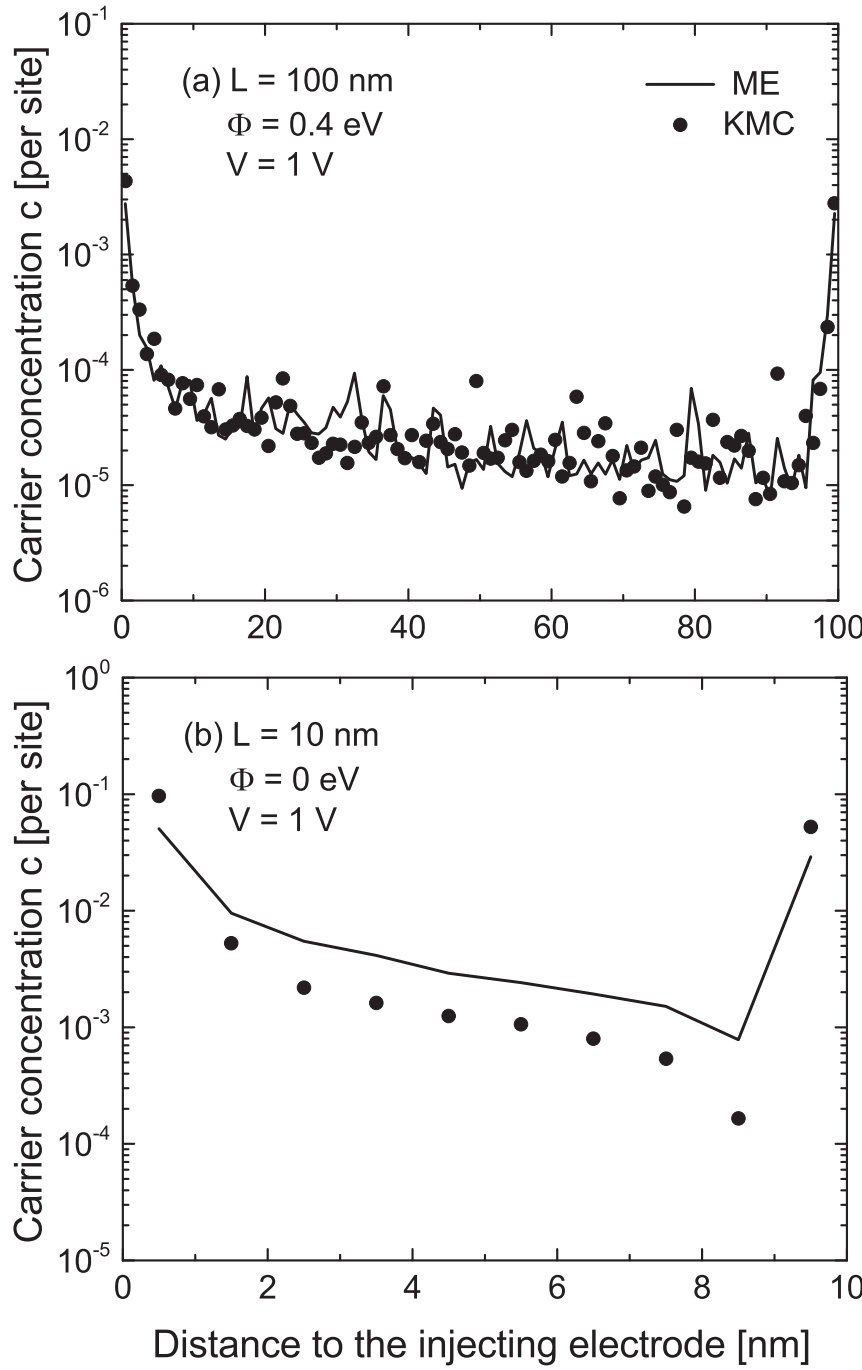

FIG. 5. Simulated carrier concentration profiles for symmetric unipolar devices with $\sigma=0.1 \mathrm{eV}$ at $T=290 \mathrm{~K}$. (a) 100 -nm-thick device with an injection barrier $\Phi=0.4 \mathrm{eV}$, studied at a voltage $V=1 \mathrm{~V}$. (b) 10 -nm-thick device with $\Phi=0 \mathrm{eV}$, at $V=1 \mathrm{~V}$. Solid curves: ME results. Symbols: KMC results. The displayed carrier concentration is equal to the laterally averaged site occupation probability.

a $100-\mathrm{nm}$ device at $1 \mathrm{~V}$. There is no significant difference between the profiles. In almost the entire device, the chargecarrier concentration is smaller than $10^{-4}$. Consistent with the simulation results discussed in Sec. III, no significant effect of Coulomb correlation is observed. We note that the charge-carrier concentration in the layer adjacent to the electrodes is actually larger than the value of about $1.8 \times 10^{-4}$ that is expected under near-thermal-equilibrium conditions when the image-charge interaction is neglected. Including the image-charge effect thus increases in this case the carrier concentration in the interface layer by more than one order of magnitude. Somewhat unexpectedly, $J_{\mathrm{ME}}$ is for thin devices and large device-averaged fields smaller than $J_{\mathrm{KMC}}$. We tentatively explain this as a "declotting" effect, illustrated in Fig. 7 of Ref. [25]. In the absence of Coulomb correlation, clots of carriers in energetically low-lying sites can occur. The 
Coulomb interaction causes a declotting by the repulsion of carriers, so that new pathways open up and the carriers are more free to move.

For devices with smaller injection barriers $(0.2$ and 0 $\mathrm{eV})$, Coulomb correlation can significantly reduce the current density. The reduction is stronger at lower voltages and smaller thicknesses, consistent with the results discussed in Sec. III. For example, for a realistic 50-nm-thick device with a $0.2 \mathrm{eV}$ injection barrier at $V=0.5 \mathrm{~V}$, the reduction of the current density by Coulomb correlation is around a factor of 2 . For 10 -nm-thick devices at $0.1 \mathrm{~V}$ the reduction is already a factor of 4. In Fig. 5(b), the charge-carrier concentration profiles for the case of $\Phi=0 \mathrm{eV}, L=10 \mathrm{~nm}$, and $V=1 \mathrm{~V}$ are shown. The results obtained from the ME and KMC simulations are significantly different. The mobility reduction is largest near the electrodes, where the charge-carrier concentration is largest, so that when including Coulomb correlation the carrier concentration increases near the electrodes and decreases in the bulk of the organic layer.

One may ask whether the parametrization scheme Eqs. (1)(3) can be directly incorporated into a one-dimensional driftdiffusion model. However, this is not immediately obvious. First, the Coulomb interaction is a long-range interaction. As the parametrization scheme is obtained for a uniform carrier concentration and electric field, it is not a priori clear whether the results will hold for a device, in which the carrier concentration and electric field can locally change abruptly (Fig. 5). Second, when the carrier concentration is around $10^{-2}$, the carrier relaxation process is very slow. It is possible that the carrier relaxation time exceeds the carrier transit time over a thin device. While the parametrization scheme applies to a fully relaxed carrier mobility, carriers in a thin device may actually not be relaxed.

\section{B. Comparison with experiment}

To investigate the relevance of the Coulomb correlation in realistic devices, we use the simulation approach developed in this work to analyze the hole-only current density-voltage characteristics of ultrathin sandwich-type devices with regioregular poly(3-hexylthiophene) (P3HT) layers in between gold electrodes measured recently by Wilbers et al. [44]. The authors demonstrated that by spin-coating P3HT layers on a gold bottom electrode, followed by gently contacting a patterned gold top electrode using a wedging transfer technique, devices can be fabricated reliably with P3HT layer thicknesses down to $5 \mathrm{~nm}$. They found that drift-diffusion simulations, which do not account for Coulomb correlation, describe the experimental current density-voltage characteristics of thick $(100 \mathrm{~nm})$ devices well, but fail to describe those of very thin (5 and $10 \mathrm{~nm}$ ) devices.

In Fig. 6, we compare the experimental current densityvoltage characteristics obtained for 100-, 40-, 10-, and 5-nm thick devices with the results of $\mathrm{ME}$ and $\mathrm{KMC}$ simulations. The simulation parameters are $a=1 \mathrm{~nm}, \lambda=0.33 \mathrm{~nm}, \sigma=$ $0.077 \mathrm{eV}, \Phi=0 \mathrm{eV}$, and $v_{1}=1.5 \times 10^{10} \mathrm{~s}^{-1}$. The relative dielectric constant has been taken equal to the measured value [45]: $\varepsilon_{\mathrm{r}}=4.4$. The other parameters were obtained by fitting the experimental results obtained for the $100-\mathrm{nm}$ devices to $\mathrm{ME}$ results. As the value of the wave function decay length $\lambda$ is relatively large, we consider in the simulations hopping to 124 neighbors. The injection barriers at both electrodes are taken equal, consistent with the analysis of the experimental data given in Ref. [44]. In the figure, we have included all available experimental data that were obtained for devices with the same circular surface area, with a diameter of $2 \mu \mathrm{m}$. There are three nominally identical 100-nm devices (open squares, triangles, and diamonds), two nominally identical 40- and 10-nm devices (open squares and triangles), and there is one 5-nm device (open squares). The simulation parameters are kept equal for all layer thicknesses.

For the 100-nm devices, the ME and KMC simulations yield only slightly different current density-voltage curves. The somewhat better overall agreement of the ME simulation results with experiment is not unexpected since the parameters are only optimized for the ME simulations. The difference between both approaches is significantly larger for the thinner devices. Although, for the 40-nm devices, the ME and KMC simulation results differ at low voltages already by about half an order of magnitude, it is, in view of the experimental sample-to-sample variation for this case, not yet possible to conclude that the KMC simulations provide a better description. However, for the 5- and 10-nm devices, the KMC simulations do provide a significantly better overall description than the ME simulations, although the agreement is not perfect. The simulations overestimate the temperature dependence of the characteristics. Beyond parameter optimization, there may be additional causes. Firstly, the actual morphology of the polymer may, at such small thicknesses, be more important for charge transport, so that the lattice model used in this work is not fully adequate. Secondly, when decreasing the organic layer thickness, the current densityvoltage characteristics become more sensitive to the charge injection and collection processes at the electrode interfaces, which may not be described to a sufficient level of accuracy. The smaller temperature dependence of the current density for small device thicknesses might indicate that the role of longer-distance temperature-independent tunneling processes becomes more significant [44]. Nevertheless, we conclude from our analysis that Coulomb correlation can indeed have a significant effect on current density-voltage characteristics of thin sandwich-type devices.

\section{SUMMARY AND CONCLUSIONS}

We have studied charge transport in organic semiconductors with Gaussian energetic disorder using ME and KMC simulations. The KMC simulations include Coulomb correlation, which is neglected in the ME simulations. When the chargecarrier concentration is above $10^{-3}$ per molecular site and the electric field is smaller than $10^{8} \mathrm{~V} / \mathrm{m}$, the ME approach is found to lead to a significantly larger charge-carrier mobility than the KMC approach. The reduction of the mobility due to Coulomb correlation is found to be fairly well described using a simple empirical parametrization scheme. Further theoretical studies are required to develop the physical basis for this parametrization scheme and will possibly provide a refinement.

For sandwich-type devices, we find that ME modeling can predict the thick devices well, but can lead to significant errors 

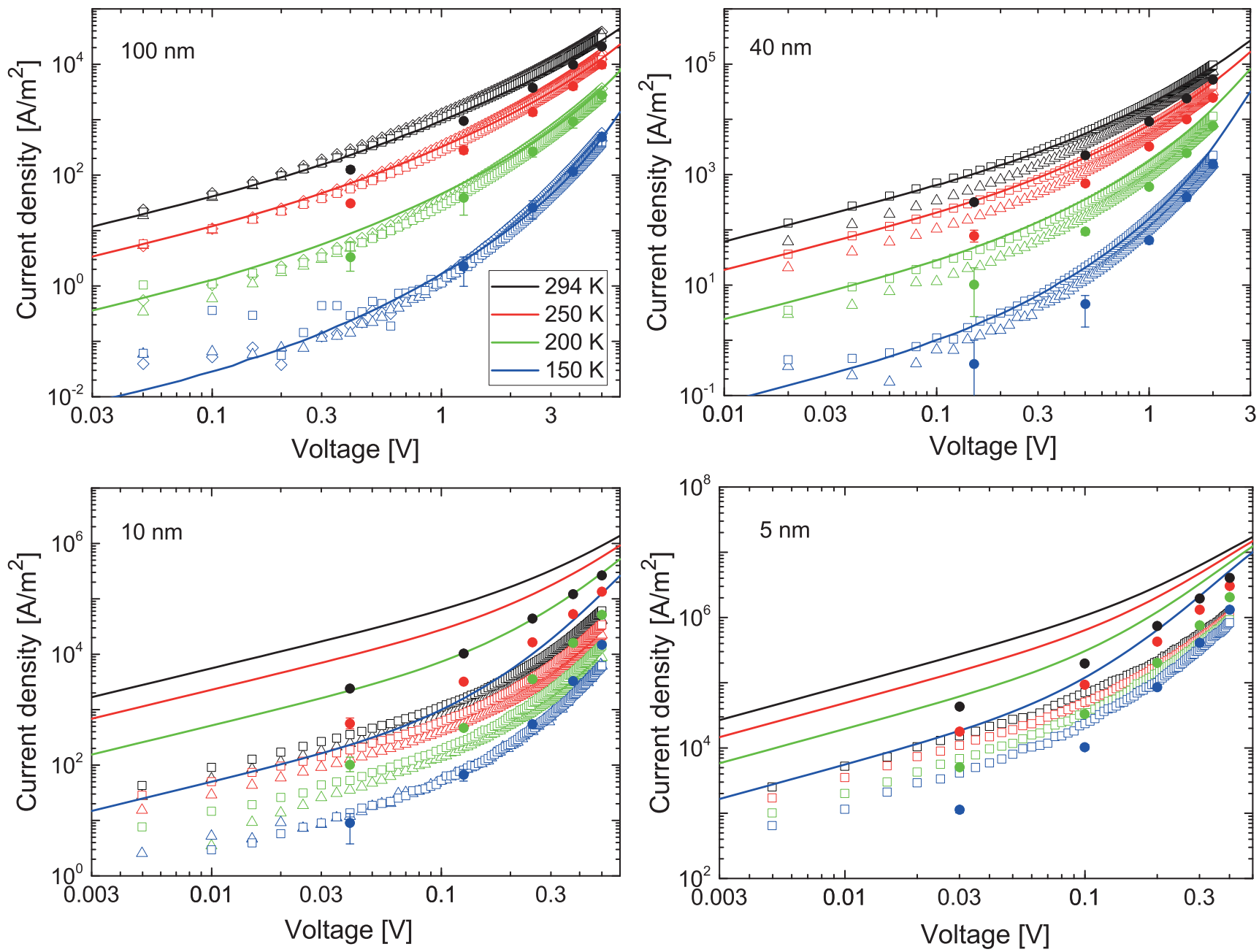

FIG. 6. Current density-voltage characteristics for P3HT devices, for various active layer thicknesses and temperatures. The open symbols are the experimental data. Open squares, triangles, and diamonds are characteristics of different samples fabricated under nominally identical conditions. The data given by the open squares are also shown in Fig. 4 of Ref. [44]. Solid curves: ME results. Filled symbols: KMC results.

in the current density and carrier concentration profile, in particular for thin devices with small injection barriers at low voltages. From a comparison of ME and KMC simulation results with experimental current density-voltage characteristics of P3HT-based unipolar devices, we conclude that Coulomb correlation can indeed be of significant importance under realistic circumstances.

\section{ACKNOWLEDGMENTS}

This work is part of the research program 3D Master Equation Simulations for Next-Generation OLEDs (MENGO), Project No. 16KIEMH01, which is jointly financed by the Netherlands Organization for Scientific Research (NWO) and Simbeyond B.V. This research is also part of the Horizon
2020 EU Projects EXTMOS (Contract No. 646176) and MOSTOPHOS (Contract No. 646259), of the German Project UNiverselles Verständnis der Defekte in Materialien für die flexible ELektronik (UNVEiL), funded by the German Federal Ministry of Education and Research (BMBF), and of the Dutch-German Project MODEOLED (Modeling of organic light-emitting diodes: From molecule to device), funded on the Dutch side by the Dutch Technology Foundation (STW; Project No. 12200), which is part of the Dutch Science Foundation (NWO), and on the German side by the Deutsche Forschungsgemeinschaft (DFG; Project No. WE1863/22-1). This work was also financially supported by the NWO-nano (STW) Program, Grant No. 11470 (W.G. van der Wiel) and the China Scholarship Council (Grant No. 201206090154). We thank Dr. Siebe van Mensfoort and Xander de Vries for stimulating discussions.
[1] V. Coropceanu, J. Cornil, D. A. da Silva Filho, Y. Olivier, R. Silbey, and J.-L. Brédas, Chem. Rev. 107, 926 (2007).

[2] H. Bässler, Phys. Stat. Sol. B 175, 15 (1993).
[3] H. Sirringhaus, P. J. Brown, R. H. Friend, M. M. Nielsen, K. Bechgaard, B. M. W. Langeveld-Voss, A. J. H. Spiering, R. A. J. Janssen, E. M. Meijer, P. Herwig, and D. M. de Leeuw, Nature 401, 685 (1999). 
[4] P. W. M. Blom, V. D. Mihailetchi, L. J. A. Koster, and D. E. Markov, Adv. Mater. 19, 1551 (2007).

[5] A. Massé, P. Friederich, F. Symalla, F. Liu, R. Nitsche, R. Coehoorn, W. Wenzel, and P. A. Bobbert, Phys. Rev. B 93, 195209 (2016).

[6] V. Rühle, A. Lukyanov, F. May, M. Schrader, T. Vehoff, J. Kirkpatrick, B. Baumeier, and D. Andrienko, J. Chem. Theory Comput. 7, 3335 (2011).

[7] P. Kordt, J. J. M. van der Holst, M. A. Helwi, W. Kowalsky, F. May, A. Badinski, C. Lennartz, and D. Andrienko, Adv. Funct. Mater. 25, 1955 (2015).

[8] P. Friederich, F. Symalla, V. Meded, T. Neumann, and W. Wenzel, J. Chem. Theory Comput. 10, 3720 (2014).

[9] F. Liu, A. Massé, P. Friederich, F. Symalla, R. Nitsche, W. Wenzel, R. Coehoorn, and P. A. Bobbert, Appl. Phys. Lett. 109, 243301 (2016).

[10] A. Massé, P. Friederich, F. Symalla, F. Liu, V. Meded, R. Coehoorn, W. Wenzel, and P. A. Bobbert, Phys. Rev. B 95, 115204 (2017).

[11] H. Uratani, S. Kubo, K. Shizu, F. Suzuki, T. Fukushima, and H. Kaji, Sci. Rep. 6, 39128 (2016).

[12] L. Wang, G. Nan, X. Yang, Q. Peng, Q. Li, and Z. Shuai, Chem. Soc. Rev. 39, 423 (2010).

[13] J. S. Bonham and D. H. Jarvis, Aust. J. Chem. 31, 2103 (1978).

[14] S. L. M. van Mensfoort and R. Coehoorn, Phys. Rev. B 78, 085207 (2008).

[15] P. S. Davids, I. H. Campbell, and D. L. Smith, J. Appl. Phys. 82, 6319 (1997).

[16] B. K. Crone, P. S. Davids, I. H. Campbell, and D. L. Smith, J. Appl. Phys. 87, 1974 (2000).

[17] F. Liu, P. P. Ruden, I. H. Campbell, and D. L. Smith, J. Appl. Phys. 111, 094507 (2012).

[18] E. Knapp, R. Häusermann, H. U. Schwarzenbach, and B. Ruhstaller, J. Appl. Phys. 108, 054504 (2010).

[19] Z. G. Yu, D. L. Smith, A. Saxena, R. L. Martin, and A. R. Bishop, Phys. Rev. B 63, 085202 (2001).

[20] J. A. Freire and G. Voss, J. Chem. Phys. 122, 124705 (2005).

[21] W. F. Pasveer, J. Cottaar, C. Tanase, R. Coehoorn, P. A. Bobbert, P. W. M. Blom, D. M. de Leeuw, and M. A. J. Michels, Phys. Rev. Lett. 94, 206601 (2005).

[22] J. J. M. van der Holst, M. A. Uijttewaal, B. Ramachandhran, R. Coehoorn, P. A. Bobbert, G. A. de Wijs, and R. A. de Groot, Phys. Rev. B 79, 085203 (2009).
[23] N. Tessler, Y. Preezant, N. Rappaport, and Y. Roichman, Adv. Mater. 21, 2741 (2009).

[24] J. Zhou, Y. C. Zhou, J. M. Zhao, C. Q. Wu, X. M. Ding, and X. Y. Hou, Phys. Rev. B 75, 153201 (2007).

[25] J. J. M. van der Holst, F. W. A. van Oost, R. Coehoorn, and P. A. Bobbert, Phys. Rev. B 83, 085206 (2011).

[26] I. A. Howard, F. Etzold, F. Laquai, and M. Kemerink, Adv. Energy Mater. 4, 1301743 (2014).

[27] N. J. van der Kaap and L. J. A. Koster, J. Comp. Phys. 307, 321 (2016).

[28] M. Mesta, M. Carvelli, R. J. de Vries, H. van Eersel, J. J. M. van der Holst, M. Schober, M. Furno, B. Lüssem, K. Leo, P. Loebl, R. Coehoorn, and P. A. Bobbert, Nat. Mater. 12, 652 (2013).

[29] A. Sharma, F. W. A. van Oost, M. Kemerink, and P. A. Bobbert, Phys. Rev. B 85, 235302 (2012).

[30] Y. Xia, W. Xie, P. P. Ruden, and C. D. Frisbie, Phys. Rev. Lett. 105, 036802 (2010)

[31] C. C. Yu, Phys. Rev. Lett. 82, 4074 (1999).

[32] J. J. M. van der Holst, F. W. A. van Oost, R. Coehoorn, and P. A. Bobbert, Phys. Rev. B 80, 235202 (2009).

[33] J. Cottaar, R. Coehoorn, and P. A. Bobbert, Org. Electron. 13, 667 (2012)

[34] A. Miller and E. Abrahams, Phys. Rev. 120, 745 (1960).

[35] R. Coehoorn, W. F. Pasveer, P. A. Bobbert, and M. A. J. Michels, Phys. Rev. B 72, 155206 (2005).

[36] Bumblebee, provided by Simbeyond B.V., available at simbeyond.com.

[37] A. Massé, R. Coehoorn, and P. A. Bobbert, Phys. Rev. Lett. 113, 116604 (2014).

[38] A. L. Efros, J. Phys. C: Solid State Phys. 9, 2021 (1976).

[39] A. L. Efros and B. I. Shklovskii, J. Phys. C: Solid State Phys. 8, L49 (1975).

[40] J. Bergli, A. M. Somoza, and M. Ortuño, Phys. Rev. B 84, 174201 (2011).

[41] E. R. Grannan and C. C. Yu, Phys. Rev. Lett. 71, 3335 (1993).

[42] A. V. Nenashev, J. O. Oelerich, A. V. Dvurechenskii, F. Gebhard, and S. D. Baranovskii, Phys. Rev. B 96, 035204 (2017).

[43] See Supplemental Material at http://link.aps.org/supplemental/ 10.1103/PhysRevB.96.205203 for more simulation results of the charge-carrier mobility.

[44] J. G. E. Wilbers, B. Xu, P. A. Bobbert, M. P. de Jong, and W. G. van der Wiel, Sci. Rep. 7, 41171 (2017).

[45] Y. S. Cho and R. R. Franklin, Trans. Electr. Electron. Mater. 13, 237 (2012). 